Paediatr.Paedolog. 2020 55 (Suppl3):S157-S169 https://doi.org/10.1007/s00608-020-00818-w

c Der/die Autor(en) 2020

\section{Till Voigtländer}

Universitätsklinik für Neurologie, Abteilung für Neuropathologie und Neurochemie, Medizinischer Universitätscampus Wien und Nationales Büro für die Umsetzung und Weiterentwicklung des NAP.se, Medizinische Universität Wien, Wien, Österreich

\title{
Zukunftsperspektiven des Nationalen Aktionsplans für seltene Erkrankungen
}

\section{Die Crux mit den Zukunftsperspektiven}

Über die Zukunftsperspektiven des Nationalen Aktionsplans für seltene Erkrankungen (NAP.se) - wie im allgemeinen jedes anderen nationalen Aktionsplans auch - zu reden oder zu schreiben, ist in mehrerlei Hinsicht ein schwieriges und riskantes Unterfangen. Ein schwieriges, da ein nationaler Aktionsplan mit seinen zahlreichen Maßnahmen einen Umfang besitzt, der in einem einzigen Beitrag nicht annähernd reflektiert werden kann. Schwierig auch, weil er die Lebensund Arbeitsbereiche vieler Menschen mit ihren individuellen Vorstellungen und Wünschen betrifft und so ein breit gefächertes Spektrum an Erwartungshaltungen kreiert. Wenn diese Erwartungshaltungen dann mit den Rahmenbedingungen und Realitäten bei der Umsetzung der Maßnahmen in Konflikt geraten und ein Vortragender oder Verfasser dies auch so zur Kenntnis bringen muss, sind Enttäuschungen bei der Zuhörerund Leserschaft vorprogrammiert. Riskant ist das Unterfangen, da der Begriff Zukunftsperspektiven per se nicht nur kurzfristige Überlegungen zu den nächsten Wochen und Monaten umfasst, sondern eine weiter in die Zukunft reichende Projektion beinhaltet, die in zeitliche Gefilde reicht, für die auf politischer Ebene - auch zum Erhalt einer notwendigen Handlungsflexibilität - häufig noch keine konkreten Planungen erstellt werden (der konkrete Planungshorizont beim NAP.se bewegt sich derzeit in Zeiträumen von 1 bis 2 Jahren). Die aktuell über uns her- eingebrochene Corona-Pandemie mit ihren manifesten Auswirkungen schon auf kurzfristige Vorhaben hat dies auf eine drastische und paradigmatische Weise offengelegt wie wenige Ereignisse zuvor. Ein Teil der im nachfolgenden Beitrag ausgeführten Zukunftsperspektiven besteht daher auch aus Szenarien, die aus den ursprünglichen Zielsetzungen und dem bisherigen Entwicklungsverlauf bei der Umsetzung des NAP.se sowie aus begleitenden europäischen Entwicklungen abgeleitet wurden, aber auf gesundheitspolitischer Ebene bislang weder diskutiert noch beschlossen wurden. Man kann diese Elemente als Visionen sehen, deren Umsetzung aus Sicht der seltenen Erkrankungen mittel- oder längerfristig sehr erstrebenswert erscheint. Aber auch eine gute Vision garantiert noch keine Umsetzung, denn die politische Entscheidungsebene mag - und sei es geleitet von anderen Sachzwängen - die Relevanz einer Umsetzung anders bewerten. Auch ist es möglich, dass zukünftige Entwicklungen die ursprüngliche Idee überholen und sie dadurch überflüssig machen. Das Schlusskapitel dieses Beitrags, das nach den vorangehenden Abschnitten mit einer kurzen Einleitung in den nationalen Aktionsplan und einer auf die Zentrenthematik fokussierten Darstellung seines aktuellen Umsetzungsstands den weiteren Zukunftsperspektiven gewidmet ist, wird gezielt auf diese Gradwanderung eingehen.

\section{Der NAP.se im Kurzportrait}

Die Geschichte des österreichischen NAP.se begann 2007/2008 als akademische Initiative mit einer vom österreichischen Orphanet-Team ${ }^{1}$ verfassten und von zahlreichen Selbsthilfegruppen unterstützten und letztlich erfolgreichen Petition für einen entsprechenden Aktionsplan. Diese wurde der damaligen Gesundheitsministerin am 29.02.2008 am ersten in ganz Europa gefeierten Tag der seltenen Erkrankungen (symbolisch bewusst auf einen seltenen Schalttag gelegt) überreicht. Nach weiteren, und politisch natürlich schwergewichtigeren, Initiativen und Entwicklungen auf europäischer Ebene einschließlich der Verabschiedung einer entsprechenden Mitteilung und einer Empfehlung [1, 2] folgten 5 Jahre (2009-2013), in denen der Aktionsplan zunächst weiter als akademisches Projekt und ab 2011 in der Nationalen Koordinationsstelle für seltene Erkrankungen (einem Kooperationsprojekt von Medizinischer Universität Wien und der Gesundheit Österreich $\mathrm{GmbH}$ ) in Zusammenarbeit mit einer zunehmenden Zahl an Akteuren aus letztlich allen relevan-

\footnotetext{
1 Orphanet (www.orpha.net), ein Gemeinschaftsprojekt der Europäischen Kommission und zahlreicher europäischer sowie einzelner außereuropäischer Länder, ist die weltweit umfangreichste Referenzdatenbank für praktisch alle Themenbereiche der seltenen Erkrankungen. Der Stammsitz befindet sich am INSERM in Frankreich, während in den Partnerländern nationale Länderteams die lokale Datenerhebung übernehmen.
} 
ten Gesundheitsbereichen entwickelt und erarbeitet wurde [3]. Nach vielen weiteren Konsultationen und Feedbackrunden mit allen involvierten Akteuren und Institutionen wurde er schließlich am 28.02.2015 als interministerielle $\mathrm{Pu}$ blikation von den Bundesministerien für Gesundheit, für Arbeit und Soziales und für Wissenschaft, Forschung und Wirtschaft veröffentlicht [4].

Der NAP.se umfasst 9 Handlungsfelder, in denen 46 Ziele definiert werden, die durch die Implementierung von $82 \mathrm{Maßnahmen}$ erreicht werden sollen (für eine gekürzte Zusammenfassung s. - Tab. 1). Die Umsetzung, die zu Beginn in zentralen Bereichen viele Vorbereitungen auf gesundheitsplanerischer Ebene erforderte, wurde zunächst von 2015-2018 von der bereits erwähnten Nationalen Koordinationsstelle im Auftrag des Gesundheitsministeriums in Angriff genommen. Nachdem allerdings die planerischen und verfahrenstechnischen Voraussetzungen geschaffen waren, verschob sich der Arbeitsschwerpunkt zunehmend in den medizinischakademischen Expertisebereich, sodass das weitere Roll-out des Aktionsplans nun seit 2019 über eine neue Einrichtung, das Nationale Büro für die Umsetzung und Weiterentwicklung des NAP.se (kurz NB-NAP.se) getragen wird. Hierbei handelt es sich wieder um ein universitäres Projekt, das bislang über einjährige Projektverträge vom Gesundheitsministerium finanziert wird.

Ein wichtiger Punkt sei noch hervorgehoben: der NAP.se war ursprünglich ganz im Sinn eines Aktionsplans, wie ihn die EU sich wünschte [2] - als Plan mit einem definierten Zeitrahmen von 2014 bis 2018 versehen. Doch bereits in der Phase der Ausarbeitung des NAP.se zeigte sich, dass aufgrund der teils raschen Entwicklungen im Bereich der seltenen Erkrankungen ein einfacher Plan einen zu starren Rahmen bilden könnte, dessen mangelnde Flexibilität zukünftigen Anforderungen nicht gerecht zu werden drohte. So wurde bereits damals festgelegt, den Aktionsplan als eine Kombination aus Plan und Strategie anzulegen, offen für eine rasche Adaptierung an neue Entwicklungen durch Abwandlung entsprechender oder Einführung neuer

Paediatr. Paedolog. 2020 · 55 (Suppl 3):S157-S169 https://doi.org/10.1007/s00608-020-00818-w (c) Der/die Autor(en) 2020

\section{T. Voigtländer}

\section{Zukunftsperspektiven des Nationalen Aktionsplans für seltene Erkrankungen}

\section{Zusammenfassung}

Im Jahr 2008 wurde das Projekt Nationaler Aktionsplan für seltene Erkrankungen (NAP.se) mit einer Petition an das Gesundheitsministerium gestartet. Im Jahr 2020, 12 Jahre später, befinden wir uns mitten in der Umsetzung dieses Plans, der mittlerweile seinen Charakter hin zu einer längerfristigen Strategie gewechselt hat. Einen zentralen Schwerpunkt bildet dabei die Umsetzung des auf die besonderen Anforderungen Österreichs zugeschnittenen Drei-StufenModells für spezialisierte Zentren für seltene Erkrankungen und der damit verbundene Anschluss an die 24 Europäischen Referenznetzwerke. Der nachfolgende Beitrag referiert zunächst den aktuellen Umsetzungsstand

und skizziert dann in 3 Abschnitten die kurzfristigen, gesundheitspolitisch bereits abgesicherten, die mittelfristigen, in einer ersten politischen Diskussion befindlichen, und die langfristigen, teils noch vagen bzw. visionären und nicht politisch diskutierten, aber interessanten und in einzelnen europäischen Nachbarländern exemplarisch umgesetzten Zukunftsperspektiven bei der weiteren Umsetzung des NAP.se.

Schlüsselwörter

Assoziiertes Nationales Zentrum · Expertisezentrum · Europäische Referenznetzwerke . Ganzheitliche Betreuung · Kodierung (seltener Erkrankungen)

\section{Future Perspectives of the National Action Plan for Rare Diseases}

\section{Abstract}

In 2008, the project to implement a national plan of action for rare diseases (NAP.se) started with a petition to the Federal Ministry of Health. Twelve years later, in 2020, we are now in the middle of the implementation process of this plan, whose character has changed in the meantime into a long-term strategy. A central priority comprises the implementation of the Three-Stage-Model of specialized centres for rare diseases designed to meet the special requirements in Austria, as well as the associated integration of these centres into the 24 European reference networks. The following article first summarizes the current state of implementation, and then outlines in three sections, the short-term prospects for the further implementation of the NAP.se, which are already secured in terms of health policy; the medium-term prospects, some of which are in initial political discussions; and the long-term prospects, some of which are still vague and/or visionary and not politically discussed, but which are interesting and have been implemented in individual neighbouring European countries in an exemplary way.

\section{Keywords}

Associated National Centre - Centre of Expertise (offizieller EU-Begriff) · European reference networks - Holistic care - Coding (of rare diseases)
Maßnahmen - und offen für eine Weiterentwicklung nach 2018. Dieser Grundcharakter spiegelt sich auch in der $\mathrm{Na}$ mensgebung des NB-NAP.se wider - und er zeigt sich im kontinuierlichen Commitment des Gesundheitsministeriums, $\mathrm{zu}$ einer erfolgreichen Umsetzung des NAP.se im Rahmen des finanziell Möglichen beizutragen.

\section{Bisherige Umsetzung des NAP.se - der Stand bis Ende 2019}

Mit Stand Ende 2019 waren 46 Maßnahmen des NAP.se, die sich auf alle 9 Handlungsfelder verteilen, teilweise oder vollständig umgesetzt. Einen zentralen Schwerpunkt hierbei bildeten die Arbeiten zum 3-stufigen Zentrenmodell in Österreich. Dieses Modell umfasst mit den Begriffen Typ A-Zentrum/Expertisecluster, Typ B-Zentrum/ Expertisezentrum und Typ C-Zentrum/ 
Tab. 1 Handlungsfelder des Nationalen Aktionsplans für seltene Erkrankungen mit Auflistung ausgewählter, teils bereits umgesetzter, teils in Umsetzung befindlicher und teils noch umzusetzender Maßnahmen

\section{Handlungs- Titel des Handlungsfelds mit Auflistung ausgewählter Maßnahmen}

feld

1

Abbildung der seltenen Erkrankungen im Gesundheits- und Sozialsystem

Entwicklung eines Kodierungssystems für SE und Implementierung in Typ A-, Typ B- und Typ C-Zentren

Prüfung der derzeitigen Abrechnungs- und Honorierungskataloge im Hinblick auf das Leistungsgeschehen im Zusammenhang mit SE

Erstellen eines Konzepts zur Einführung einer Patienteninformationskarte Verbesserung der medizinisch-klinischen Versorgung der von SE Betroffenen

Ausarbeitung von spezifischen Leistungs- und Qualitätskriterien für Typ A-, Typ Bund Typ C-Zentren

Entwicklung und Durchführung von Designationsverfahren für Typ A-, Typ B- und Typ C-Zentren

Entwicklung eines Evaluationsverfahrens für Typ A- und Typ B-Zentren; Abstimmung mit korrespondierenden Verfahren auf EU-Ebene

Unterstützung der horizontalen und transsektoralen Vernetzung der Tyр A-, Тур B- und Typ C-Zentren mit den anderen Versorgungsstufen

Unterstützung bei der europäischen Vernetzung mit ERN
Verbesserung der Diagnostik von SE

Definition von Leistungs- und Qualitätskriterien für medizinische Laboratorien im Bereich der SE

Entwicklung eines Bewerbungs-, Begutachtungs- und Designationsverfahrens für mit der Diagnostik von SE befasste medizinische Laboratorien

Ausarbeitung von Kompetenzkriterien für Expertinnen und Experten, die mit der apparativen Diagnostik von SE befasst sind

Einrichtung eines ständigen wissenschaftlichen Beirats für das österreichische Neugeborenenscreening

Verbesserung der Therapie und des Zugangs zu Therapien für von SE Betroffene

Teilnahme an europäischen Kooperationsprojekten zur Sicherung der nachhaltigen Finanzierung von "orphan drugs" (z. B. MoCA-OMP)

Preiserhebung von ausgewählten „orphan drugs“ im intramuralen sowie niedergelassenen Bereich

Etablieren optimierter Versorgungsabläufe am Best Point of Service

Etablieren eines regelmäßigen Austauschs zwischen Chefärztinnen bzw. Chefärzten, der sozialen Krankenversicherung und der Patientenschaft

Förderung der Forschung im Bereich SE

Designation von Expertisezentren mit einem Schwerpunkt auf Forschung

Teilnahme Österreichs an ERN bezüglich Forschungsaspekten

Verbesserung des Wissens über und des Bewusstseins zu SE

Vermitteln von Basiswissen zu SE und von damit verbundenen möglichen Behandlungsoptionen

Spezifische qualitätsgesicherte Fort- und Weiterbildungsmaßnahmen für Ärztinnen und Ärzte sowie weitere Gesundheitsberufe

Ergänzen eines Schwerpunkts zu SE im Gesundheitsportal und Bereitstellen qualitätsgesicherter Informationen zu SE über Orphanet Austria

Verbesserung der epidemiologischen Kenntnisse im Kontext SE

Bestandsaufnahme bestehender Patientenregister für SE

Definition und Abstimmung (datenschutz-)rechtlicher, struktureller und finanzieller Rahmenbedingungen sowie von Bestimmungen zur Qualitätssicherung

Sicherstellen der Interoperabilität mit bestehenden relevanten nationalen und internationalen Patientenregistern und Datenerfassungssystemen

Entwickeln eines minimalen Datensatzes unter Berücksichtigung internationaler Entwicklungen
Assoziiertes Zentrum 3 Differenzierungsstufen für hochspezialisierte Versorgungseinrichtungen für Patientinnen und Patienten mit einer seltenen Erkrankung, die einerseits die vorhandene Spezialexpertise für seltene Erkrankungen in vielen medizinischen Bereichen in Österreich unter Berücksichtigung der jeweils zur Verfügung stehenden personellen und strukturellen Rahmenbedingungen abbilden sollen und andererseits im Einklang mit den hierfür auch auf europäischer Ebene entwickelten relevanten Qualitätsstandards und -definitionen stehen [3, 4]. Das Expertisezentrum oder Typ B-Zentrum steht dabei im Mittelpunkt des österreichischen Modells und entspricht dem auf europäischer Ebene definierten „Center of Expertise“ des European Committee of Experts on Rare Diseases (EUCERD²; [5]). Zu seinen zentralen Leistungs- und Qualitätskriterien gehören die durch detaillierte Fallzahlen aus den letzten 3 Jahren dokumentierte LeuchtturmExpertise für eine definierte Gruppe seltener Erkrankungen aus dem Fachgebiet der jeweiligen Einrichtung, die Existenz eines multiprofessionellen Versorgungsansatzes für diese Krankheitsbilder, der dem aktuellen Stand des medizinischen Wissens und entsprechenden Vorgaben aufEU-Ebene entspricht, und die Verfügbarkeit von mindestens 2 Fachärztinnen bzw. Fachärzten mit vergleichbarer Spezialexpertise für diese Erkrankungen, die zusammen mindestens $75 \%$ ihrer Arbeitszeit ausschließlich diesem Bereich widmen. Mit Spezialexpertise ist dabei ein klinisches Wissens- und Erfahrungsniveau gemeint, dass sich oberhalb jenes grundsätzlichen Wissens- und Kenntnisniveaus bewegt, das für tertiäre Einrichtungen wie Universitätskliniken charakteristisch ist (daher der Begriff des Leuchtturms). Und die Dupli- bis Multiplikation der fachärztlichen Spezialexpertise (es können auch mehr als 2 Spezialistinnen bzw. Spezialisten in diesem Bereich am Zentrum arbei-

2 EUCERD, ein aus Expertinnen und Experten der verschiedensten Bereiche sowie Vertretern aller Mitgliedstaaten zusammengesetztes Beratungsgremium der EU-Kommission in den Jahren 2011-2013. 


\begin{tabular}{|c|c|}
\hline $\begin{array}{l}\text { Handlungs- } \\
\text { feld }\end{array}$ & Titel des Handlungsfelds mit Auflistung ausgewählter Maßnahmen \\
\hline \multirow[t]{4}{*}{8} & Einrichtung ständiger Beratungsgremien für SE beim BMG \\
\hline & $\begin{array}{l}\text { Konstituieren des „Beirats für seltene Erkrankungen“ als Beirat gemäß § } 8 \text { Bun- } \\
\text { desministeriengesetz }\end{array}$ \\
\hline & Beibehalten der "Strategischen Plattform für SE“ \\
\hline & Fortsetzung der regelmäßigen Sitzungen und Arbeiten beider Gremien \\
\hline \multirow[t]{4}{*}{9} & Anerkennung der Leistungen der Selbsthilfe \\
\hline & $\begin{array}{l}\text { Gründung einer österreichweiten Dachorganisation der Selbsthilfegruppen von } \\
\text { Personen mit SE }\end{array}$ \\
\hline & $\begin{array}{l}\text { Schaffung von Rahmenbedingungen für die Sicherstellung einer langfristigen } \\
\text { Finanzierung }\end{array}$ \\
\hline & $\begin{array}{l}\text { Vertretung der Selbsthilfe in Entscheidungsgremien und Einbeziehen in Entschei- } \\
\text { dungsprozesse }\end{array}$ \\
\hline \multicolumn{2}{|c|}{$\begin{array}{l}\text { BMG Bundesministerium für Gesundheit, ERN Europäisches Referenznetzwerk, MoCA-OMP Mech- } \\
\text { anisms of Coordinated Access to Orphan Medicinal Products in Europe, SE seltene Erkrankungen }\end{array}$} \\
\hline
\end{tabular}

ten) dient nicht nur der ausreichenden Absicherung der ärztlichen zeitlichen Ressourcen (speziell in Urlaubs- und Krankheitszeiten), sondern der langfristigen und nachhaltigen Absicherung des Standorts als solchen.

Assoziierte Zentren oder Typ C-Zentren verfügen ebenfalls über eine derartige Leuchtturm-Expertise für eine definierte Gruppe seltener Erkrankungen, die über entsprechende Fallzahlen belegt ist. Im Unterschied zu Expertisezentren reicht für ein Assoziiertes Zentrum aber das Vorhandensein einer Fachärztin bzw. eines Facharztes aus, die bzw. der nachweislich über die erforderliche Spezialexpertise verfügt. Zudem darf auch das multiprofessionelle Team, das in die Versorgung der Patientinnen und Patienten involviert ist, kleiner dimensioniert sein $[3,4]$. Der wesentliche Unterschied liegt also in den personellen und strukturellen Ressourcen, nicht in der Expertise. Allerdings führen diese Abstriche bei den Ressourcen auch dazu, dass Assoziierte Zentren auf gesundheitspolitischer und Versorgungsebene nicht als nachhaltig angesehen und definiert werden. Geht der Experte bzw. die Expertin weg oder scheiden er oder sie aus dem Dienst aus, hört das Zentrum ohne geeignete Nachfolge auf $\mathrm{zu}$ existieren.

Expertisecluster oder Typ A-Zentren schließlich bestehen aus einem Zusammenschluss von mindestens 3, besser mehr Expertisezentren (Typ BZentren) aus unterschiedlichen medizi- nischen Fachgebieten - sowie fakultativ weiteren Assoziierten Zentren (Typ CZentren) - an einem Ort. Die einzelnen Mitgliedszentren müssen dann 2 Aufgaben erfüllen. Zum einen fungieren sie weiterhin für ihre jeweilige spezifische Gruppe seltener Erkrankungen mit all ihrer Expertise und ihren Strukturen als reguläres Typ B- oder Typ CZentrum. Zum anderen sollen sie ihre Expertise in eine übergeordnete Struktur einbringen, die nicht mehr die einzelnen Krankheitsgruppen betrachtet (das geschieht ja bereits auf der Ebene der Einzelzentren), sondern eine neue Dimension der Integration von Expertise über die medizinischen Fachgrenzen hinweg anstrebt und durch den fachgebietsübergreifenden Zusammenschluss des Spezialwissens eine Plattform für die Diskussion und Lösung komplexester Fälle im Bereich der seltenen Erkrankungen, insbesondere jener von Patienten bzw. Patientinnen mit unklarer oder noch völlig fehlender Diagnose, bietet $[3,4]$. Kernelemente dieser neuen Plattform könnten beispielsweise die gezielte, personell abgesicherte Einrichtung einer Koordinations- bzw. Anlaufstelle für Patienten und Patientinnen oder betreuende Fachärztinnen und Fachärzte, die Etablierung regelmäßiger gemeinsamer interdisziplinäre Fallkonferenzen (sogenannte SE-Boards) und die Einrichtung gemeinsam genutzter aufwendiger und/ oder innovativer Spezialdiagnostiken sein.
Von der Theorie in die Praxis das Designationsverfahren für Expertisezentren

Die Umsetzung dieses Zentrenmodells startete aus mehreren Gründen bewusst mit der Etablierung eines Designationsverfahrens für Expertisezentren (Typ BZentren). Die wichtigsten Gründehierfür waren folgende: Erstens steht das Konzept des Typ B-Zentrums im Mittelpunkt des Modells. Es ist die einzige Grundkomponente, bei der ein langfristiger $\mathrm{Be}$ stand der Einrichtung mit nachhaltiger Finanzierung angestrebt und - soweit dies aus heutiger Sicht möglich ist - gesichert wird. Zweitens stellen Typ B-Zentren auch das zentrale Grundelement zur Bildung von Typ A-Zentren dar - ohne ausreichende Zahl von Typ B-Zentren an einem Standort kann es einen Expertisecluster gar nicht geben. Drittens stellt ein vollständig ausgereiftes und etabliertes Designationsverfahren für Typ B-Zentren anschließend auch eine ideale Matrize für ein analoges, hinsichtlich bestimmter Kriterien abgespecktes und vereinfachtes Verfahren für die Designation von Typ C-Zentren dar; die wesentlichen Ausarbeitungsschritte wären damit schon erledigt und es bedürfte nur noch gezielter Adaptierungen bei den Kriterien wie dem eigentlichen Verfahrensablauf. Und viertens waren Typ B-Zentren seitens der gesundheitspolitischen Ebene schon früh als ein zentrales Bindeglied zwischen der in Österreich vorhandenen Expertise und derjenigen in den Europäischen Referenznetzwerken $(\text { ERN })^{3}$ vorgesehen [6]. Auf dieser Entscheidung beruht auch die strikte Verknüpfung der nationalen Designation als Expertisezentrum mit der formalen Unterstützung einer Einrichtung durch das

\footnotetext{
3 Bei ERN handelt es sich um Zusammenschlüsse von hochspezialisierten Einrichtungen für definierte Gruppen seltener Erkrankungen aus ganz Europa in Netzwerken, die die von ihnen betreuten seltenen Erkrankungen entweder in einem übergeordneten medizinischen Fachgebiet (z.B. ein Netzwerk für seltene Hauterkrankungen), in einem funktionellen Organsystem (z. B. ein Netzwerk für seltene Lebererkrankungen) oder in einer definierten Krankheitspathologie (z.B. ein Netzwerk für seltene Tumorerkrankungen) zusammenfassen.
} 
Gesundheitsministerium bei seiner Bewerbung um eine Vollmitgliedschaft in einem ERN. Letztere erfolgt nur, wenn eine Einrichtung zuvor national als Typ BZentrum designiert wurde bzw. alle wesentlichen Teile des Verfahrens bereits positiv durchlaufen hat.

Die Ausarbeitung des Designationsprozesses erfolgte in 4 in ihrer zeitlichen Umsetzung teilweise überlappenden Phasen und schloss Pilotprozesse und anschließende Adaptierungen und Optimierungen auf Basis der gewonnenen Erfahrungen mit ein. Diese Phasen umfassen erstens die rechtliche Verankerung der seltenen Erkrankungen und des österreichischen Zentrenmodells in der nationaler Gesundheitsplanung durch Integration in den Österreichischen Strukturplan Gesundheit, zweitens die Identifikation interessierter Einrichtungen über ein landesweites, systematisches Mapping-Verfahren mit Information aller Spitalseinrichtungen, drittens die Ausarbeitung sämtlicher Unterlagen für das Designationsverfahren und viertens die Ausarbeitung des konkreten Verfahrensablaufs einer Designation von der Auswahl der Kandidaten für eine Designationsrunde über die Beauftragung eines Verfahrens bis hin zur Gesamtbegutachtung und finalen Beschlussfassung im höchsten Entscheidungs- und Steuerungsgremium im Gesundheitsbereich, der Bundeszielsteuerungskommission. Eine Auswahl der detaillierten Handlungsschritte und der beteiligten Akteure findet sich in - Tab. 2.

Mithilfe dieses in 2 Pilotdurchgängen schrittweise verfeinerten Verfahrens wurden bis Anfang 20202 vollständige Designationsrunden, die erste mit 2, die zweite mit 7 Einrichtungen, durchgeführt und abgeschlossen, eine dritte Designationsrunde wurde Ende 2019 mit 9 neuen Kandidaten eingeleitet. Österreich verfügt damit aktuell über 9 Expertisezentren (Typ B-Zentren) für definierte Gruppen seltener Erkrankungen, die 8 medizinische Fachbereiche abdecken ${ }^{4}$, mit der Aussicht auf 9 weitere Expertise-

\footnotetext{
${ }^{4}$ Im Fachbereich der seltenen Hauterkrankungen wurden bereits 2 Zentren designiert [7].
}

zentren in naher Zukunft, die 2 weitere Fachbereiche hinzufügen werden.

Acht Fachbereiche mit Expertisezentren als Kontakt- und Knotenpunkte in nur 8 der seit 2017 existierenden 24 verschiedenen ERN hätte allerdings eine unzureichende Integration Österreichs in die europäischen Netzwerke dargestellt, ein Problem, das in unterschiedlichem Umfang nahezu alle Mitgliedstaaten mit Österreich teilten. Unter gemeinsamer Führung von Griechenland und Österreich wurde daher in dem den ERNProzess begleitenden und mitsteuernden Gremium aller EU- und EEA-Mitgliedstaaten, dem Board of Member States on European Reference Networks (BoMS), von 2017 bis 2018 ein ergänzendes Erweiterungsszenario für ERN erarbeitet und beschlossen, das es den Mitgliedstaaten erlaubt, in ERN, in denen sie nicht mit einem oder mehreren Expertisezentren als Vollmitglieder vertreten sind, eine zweite Ebene der Mitgliedschaft durch sogenannte Assoziierte Nationale Zentren (ANZ) und/oder Coordination Hubs zu eröffnen. Österreich hat bereits 2018 mit der nationalen Umsetzung dieser Möglichkeit begonnen und mithilfe eines aus dem Designationsverfahren für Typ BZentren abgeleiteten vereinfachten Verfahrens bis Herbst 201942 Einrichtungen als ANZ designiert und der EU-Kommission gemeldet, sodass es jetzt seit Ende 2019 mit insgesamt 49 Einrichtungen in allen ERN vertreten ist (• Tab. 3).

\section{Die weitere Umsetzung des NAP.se - die Zukunfts- perspektiven}

Schon eingangs des Beitrags wurde auf das problematische Verhältnis zwischen Zukunftsperspektiven und (politischer) Umsetzungsrealität verwiesen, das umso risikobehafteter ist, je weiter die Perspektive in der Zukunft liegt. Um also beim Blick in die Glaskugel - und nichts anderes sind Zukunftsperspektiven gerade jetzt in Zeiten einer Corona-Pandemie - einerseits die erforderliche Vorsicht walten zu lassen und so keine falschen Hoffnungen und Erwartungshaltungen zu wecken und andererseits dennoch einen Ausblick auf die längerfristigen Perspektiven in der weiteren
Umsetzung des NAP.se bieten zu können, untergliedert sich der nachfolgende Abschnitt in 3 Teile: (1) Die kurzfristigen, nahezu gesicherten Perspektiven der nächsten 2 Jahre, (2) die ergänzenden, mittelfristigen Optionen, abgeleitet aus der bisherigen Logik der nationalen und europäischen Entwicklungen, aber noch ohne umfassende Abstimmung mit der Ebene der Gesundheitsplanung, und (3) die langfristigen, noch vagen Optionen, in welche Richtungen eine Weiterentwicklung des NAP.se sinnvoll erscheint, ohne dass es hierzu bislang konzeptionelle Vorgespräche mit den betroffenen gesundheits- und sozialpolitischen Akteuren gegeben hätte.

\section{Zukunftsperspektiven 1 - Die nächsten Schritte auf Basis konkreter Planungen}

\section{Handlungsfeld 2 - Medizinisch- klinische Versorgung}

Diese konkreten Planungen betreffen die nächsten Schritte in den Handlungsfeldern HF 2, HF 1 und HF 3 (• Tab. 1). In HF 2 geht es dabei um die weitere Umsetzung des Zentrenmodells und hier zunächst - mit politischerseits höchster Priorität - um die Fortsetzung und den Abschluss der Designation von Expertisezentren (Typ B-Zentren), basierend auf dem Bewerberkreis, der in den damaligen Mapping-Runden durch Orphanet Austria sein dezidiertes Interesse an einer Zentrumsdesignation bekundet hat (- Tab. 2). Der politischen Leitlinie folgend, dass diese Designationsverfahren vor dem nächsten Call für neue Vollmitglieder in existierenden ERN abgeschlossen sein sollen, wurde Ende des vergangenen und Anfang dieses Jahres bereits ein detailliertes Konzept mit konkreten Zeitplanungen für 2 weitere Designationsrunden mit jeweils 10 Zentren erstellt, dessen für März/April vorgesehener Start durch die Corona-Krise ausgebremst wurde. Zu diesem Konzept gehörte - und gehört weiterhin - die Durchführung vorbereitender Diskussionsrunden (intern Roundtables getauft) mit jeweils allen Interessenten für ein definiertes ERN. Denn für diese vierte und fünfte Designationsrunde stellen sich neue Herausforderungen. Es existieren näm- 
Tab. 2 Darstellung der zentralen Phasen und wesentlichen Elemente mit den jeweils zugehörigen Handlungsschritten und Akteuren bei der Entwicklung und Implementierung eines nationalen Designationsprozesses für Expertisezentren (Typ B-Zentren) einschließlich seiner Integration in einen nationalen gesundheitsplanerischen Rechtsrahmen

\begin{tabular}{|c|c|c|}
\hline Phasen & Handlungen & Akteur bzw. Akteure \\
\hline \multirow{2}{*}{$\begin{array}{l}\text { Verankerung von SE } \\
\text { in nationaler Ge- } \\
\text { sundheitsplanung }\end{array}$} & $\begin{array}{l}\text { Ausarbeitung eines Entwurfs zur textlichen Integration der SE und des österreichischen Zentrenmo- } \\
\text { dells in das nationale Planungsinstrument ÖSG }\end{array}$ & $\begin{array}{l}\text { NKSE, GÖG, } \\
\text { Planungsgremien }\end{array}$ \\
\hline & Beschluss der textlichen Integration der SE und des österreichischen Zentrenmodells in den ÖSG & $\begin{array}{l}\text { Planungsgremien }{ }^{\mathrm{a}} \text {, } \\
\text { BZK }\end{array}$ \\
\hline \multirow[t]{5}{*}{$\begin{array}{l}\text { Identifikation von } \\
\text { Kandidaten }\end{array}$} & Umfassendes Mapping der Expertise für SE in Österreich (Bottom-up-Komponente) & $\begin{array}{l}\text { Orphanet Austria } \\
(2015)\end{array}$ \\
\hline & $\begin{array}{l}\text { Einholung und Auswertung von Interessenbekundungen für Designationsverfahren (Bottom-up- } \\
\text { Komponente) }\end{array}$ & $\begin{array}{l}\text { Kandidaten, NKSE } \\
\text { (2016) }\end{array}$ \\
\hline & $\begin{array}{l}\text { Entwicklung einer Priorisierungsstrategie und erste Reihung von Kandidaten für nachfolgende Desi- } \\
\text { gnationsrunden }\end{array}$ & $\begin{array}{l}\text { BMG, STP, NKSE } \\
\text { (2017ff.) }\end{array}$ \\
\hline & $\begin{array}{l}\text { Einholung detaillierter Leistungsinformationen von den Einrichtungen mit Interessenbekundung } \\
\text { sowie einzelnen weiteren Einrichtungen im Rahmen des Bewerbungsverfahrens für ANZ für ERN }\end{array}$ & $\begin{array}{l}\text { Kandidaten, NKSE } \\
(2018 / 2019)\end{array}$ \\
\hline & Zweite Reihung von Kandidaten für nachfolgende Designationsrunden & $\begin{array}{l}\text { BMG, STP, NKSE } \\
(2019)\end{array}$ \\
\hline \multirow{4}{*}{$\begin{array}{l}\text { Erstellen der } \\
\text { Unterlagen für die } \\
\text { Designation }\end{array}$} & $\begin{array}{l}\text { Ausarbeitung der spezifischen Leistungskriterien (operationale Kriterien) für Pilotdesignationen (Fra- } \\
\text { gebogen) }\end{array}$ & NKSE (2016) \\
\hline & Ausarbeitung der Unterlagen für externe Fachgutachter bzw. Fachgutachterinnen (Gutachterbogen) & NKSE (2016) \\
\hline & Ausarbeitung der Unterlagen für die interne Begutachtung durch NKSE & NKSE (2016) \\
\hline & Überarbeitung des Fragebogens nach Durchführung der Pilotdesignationen & NKSE (2017) \\
\hline \multirow{15}{*}{$\begin{array}{l}\text { Ablauf des Designa- } \\
\text { tionsverfahrens }\end{array}$} & Konsensuelle Vorauswahl von Einrichtungen für die Einleitung eines Designationsverfahrens & BMG, STP, NKSE ${ }^{b}$ \\
\hline & Einholung der Zustimmung der betroffenen Bundesländer (Top-down-Komponente) & NKSE $^{\mathrm{b}}$ \\
\hline & Vorstellung in den Gremien der Gesundheitsplanung ${ }^{a}$ (Top-down-Komponente) & $\begin{array}{l}\text { NKSE }^{\mathrm{b}} \\
\text { Planungsgremien }^{\mathrm{a}}\end{array}$ \\
\hline & Nach Zustimmung der Planungsgremien Einholung der weiteren Zustimmungserklärungen ${ }^{c}$ & $\mathrm{NKSE}^{\mathrm{b}}$, Kandidaten \\
\hline & $\begin{array}{l}\text { Beschluss zur Einleitung eines Designationsverfahrens, Beauftragung NKSE/NB-NAP.se (Top-down- } \\
\text { Komponente) }\end{array}$ & BZK \\
\hline & Koordination und Durchführung des Designationsverfahrens & NKSE/NB-NAP.se \\
\hline & $\begin{array}{l}\text { - Versand und Einholung der Bewerbungsunterlagen (Fragebogen plus Anlagen) einschließlich } \\
\text { Feedbackrunden }\end{array}$ & $\begin{array}{l}\text { NKSE/NB-NAP.se mit } \\
\text { Kandidaten }\end{array}$ \\
\hline & - Identifikation, Auswahl und Kontaktaufnahme externe Gutachter bzw. Gutachterinnen & $\begin{array}{l}\text { NKSE/NB-NAP.se mit } \\
\text { BMG, STP }\end{array}$ \\
\hline & - Aussendung Bewerbungs- und Gutachterunterlagen an externe Gutachter bzw. Gutachterinnen & NKSE/NB-NAP.se \\
\hline & - Durchführung interne Vor-Ort-Visite mit Erstellung von Teilgutachten & NKSE/NB-NAP.se \\
\hline & - Einholung und Auswertung externer Fachgutachten (inklusive externer Vor-Ort-Visite) & NKSE/NB-NAP.se \\
\hline & - Erstellung eines zusammenfassenden Gesamtgutachtens & NKSE/NB-NAP.se \\
\hline & - Weiterleitung des Gesamtgutachtens an BMG/Planungsgremien ${ }^{\text {a }}$ & NKSE/NB-NAP.se \\
\hline & $\begin{array}{l}\text { Entscheidungsfindung auf Ebene Planungsgremien, bei Zustimmung Beschluss zur Designation } \\
\text { durch BZK (Top-down-Komponente) }\end{array}$ & $\begin{array}{l}\text { Planungsgremien }{ }^{\mathrm{a}} \text {, } \\
\text { BZK }\end{array}$ \\
\hline & Ausstellung eines Designationsbescheids an die jeweilige Einrichtung & BMG \\
\hline \multicolumn{3}{|c|}{$\begin{array}{l}\text { ANZ Assoziierte Nationale Zentren, BMG Bundesministerium für Gesundheit, BZK Bundeszielsteuerungskommission, ERN Europäische Referenznetzwerke, } \\
\text { GÖG Gesundheit Österreich GmbH, NB-NAP.se Nationales Büro für die Umsetzung und Weiterentwicklung des NAP.se, NKSE Nationale Koordinationsstelle } \\
\text { für seltene Erkrankungen, ÖSG Österreichischer Strukturplan Gesundheit, SE seltene Erkrankungen, STP Strategische Plattform } \\
\text { 'Die Gremien der Gesundheitsplanung, kurz Planungsgremien, stellen eine aus 3-4 Ebenen bestehende, hierarchisch aufsteigende Kaskade von Beratungs- } \\
\text { und (Vor-)Entscheidungsgremien im Bereich der Gesundheitsplanung dar, die mit Vertretern bzw. Vertreterinnen der Bundesländer, der Sozialversicherungs- } \\
\text { träger und des Gesundheitsministeriums besetzt sind. Höchste und letzte Entscheidungsebene für nationale Belange ist dann die BZK } \\
\text { bab } 2019 \text { ersetzt durch das NB-NAP.se } \\
\text { 'Zu den weiteren Zustimmungserklärungen gehören diejenigen des Krankenanstaltenträgers, des Kostenträgers (falls abweichend vom Krankenanstalten- } \\
\text { träger), der Kollegialen Führung, der Geschäftsführung und, falls es um eine universitäre Einrichtung geht, des Rektorats }\end{array}$} \\
\hline
\end{tabular}


Tab. 3 Zusammenstellung aller 24 Europäischen Referenznetzwerke mit Angabe der an den jeweiligen Netzwerken teilnehmenden österreichischen Einrichtungen (linke Spalte) und ihres Designationsstatus in Österreich (rechteSpalte; modifiziert nach Unterberger [7])

Europäische Referenznetzwerke (ERN) und Mitglieder aus Österreich

Designationsstatus in Österreich

ERN BOND (ERN für seltene Knochenerkrankungen)

Expertisezentrum für Knochenerkrankungen, Störungen des Mineralhaushaltes und Wachstumsstörungen (AKH Wien, Orthopädisches Spital Speising, Hanusch Krankenhaus Wien)

ERN CRANIO (ERN für seltene und komplexe kraniofaziale Anomalien und Hals-, Nasen-, Ohrenkrankheiten) Expertisezentrum für Lippen-Kiefer-Gaumenspalten und kraniofaziale Anomalien (PMU Salzburg)

EZ

EZ

Endo-ERN (ERN für seltene endokrinologische Erkrankungen)

Zentrum für pädiatrische Endokrinologie (Klinikum Wels-Grieskirchen)

Zentrum für seltene endokrine Erkrankungen (AKH Wien)

Zentrum für seltenen Diabetes und kongenitalen Hyperinsulinismus ( $A K H$ Wien)

Zentrum für Varianten der Geschlechtsentwicklung (AKH Wien)

ERN EpiCARE (ERN für Epilepsien)

Expertisezentrum für seltene und komplexe Epilepsien (PMU Salzburg)

Pädiatrisches Epilepsie-Zentrum Wien (AKH Wien)

ERKNet (ERN für seltene Nierenerkrankungen)

Zentrum für seltene Nierenerkrankungen des Kindes- und Erwachsenenalters (Medizinische Universität Innsbruck)

Zentrum für Pädiatrische Dialyse und Nierentransplantation/Komplementerkrankungen (AKH Wien)

ERN-RND (ERN für seltene neurologische Erkrankungen)

Expertisezentrum für seltene Bewegungsstörungen Innsbruck (Medizinische Universität Innsbruck)

Zentrum für pädiatrische seltene neurologische Erkrankungen (AKH Wien)

ERNICA (ERN für seltene hereditäre und kongenitale Erkrankungen des Verdauungstraktes)

Zentrum für angeborene intestinale Malformationen (Medizinische Universität Graz)

Pädiatrisch colorectales Zentrum Linz (Kepler Universitätsklinikum Linz)

Zentrum für seltene kolorektale Erkrankungen, Inkontinenz-Erkrankungen und Erkrankungen des Beckenbodens im Kindesalter (AKH Wien)

Abteilung für Kinder- und Jugendchirurgie (Sozialmedizinisches Zentrum Ost Wien)

ERN-LUNG (ERN für Atemwegserkrankungen)

Zentrum für Pulmonale Arterielle Hypertonie (Medizinische Universität Graz)

Cystische Fibrose Zentrum der Medizinischen Universität Innsbruck (Medizinische Universität Innsbruck)

Zentrum für pulmonale Hypertension (AKH Wien)

Zentrum für seltene Lungenerkrankungen im Kindesalter (AKH Wien)

ERN-Skin (ERN seltene und undiagnostizierte Hauterkrankungen)

Expertisezentrum für Genodermatosen mit Schwerpunkt Epidermolysis bullosa (EB-Haus Salzburg)

ERN EURACAN (seltene solide Tumore des Erwachsenenalters)

Expertisezentrum für Knochen- und Weichteiltumore (Medizinische Universität Graz)

ANZ

ANZ

ANZ

ANZ

EZ

ANZ $^{\mathrm{a}}$

ANZ

ANZ

EZ

ANZ

ANZ

ANZ

ANZ

ANZ

$\mathrm{ANZ}^{\mathrm{a}}$

ANZ $^{\mathrm{a}}$

$\mathrm{ANZ}^{\mathrm{a}}$

ANZ $^{\mathrm{a}}$

EZ

EZ

ANZ

ANZ

EZ

ANZ

ANZ

ANZ

ANZ

ANZ

Klinik für Augenheilkunde und Optometrie (Kepler Universitätsklinikum Linz)

Zentrum für erbliche Netzhauterkrankungen (AKH Wien) 
ERN GENTURIS (ERN für genetisch bedingte Tumor-Risiko-Syndrome)

Zentrum für erbliche Tumorerkrankungen Innsbruck (Medizinische Universität Innsbruck)

ANZ

Zentrum für Neurofibromatose (AKH Wien)

ANZ

ERN GUARD-HEART (ERN für seltene und komplexe Herzerkrankungen)

Kardiomyopathieprogramm Innsbruck (Medizinische Universität Innsbruck)

ANZ

Kinderherzzentrum Wien (AKH Wien)

ANZ

ERN ITHACA (ERN für kongenitale Fehlbildungen und seltene geistige Beeinträchtigungen)

Zentrum für seltene angeborene Fehlbildungen und Entwicklungsstörungen (Medizinische Universität Innsbruck)

MetabERN (ERN für erbliche Stoffwechselstörungen)

Stoffwechselambulanz der Univ. Klinik für Kinder- und Jugendheilkunde Graz (Medizinische Universität Graz)

Zentrum für Angeborene Stoffwechselstörungen mit Schwerpunkt Organazidämien, Harnstoffzyklus- und Fettsäureoxidationsstörungen (Medizinische Universität Innsbruck)

Zentrum für angeborene Stoffwechselstörungen mit Schwerpunkt Mitochondriopathien (Mitocenter) und lysosomale Speicherkrankheiten (PMU Salzburg)

Zentrum für angeborene Stoffwechselerkrankungen (AKH Wien)

ERN PaedCan (ERN für pädiatrische Onkologie)

Expertisezentrum für pädiatrische Onkologie (St. Anna Kinderspital Wien)

ERN RARE-LIVER (ERN für seltene hepatologische Erkrankungen)

Zentrum für seltene Lebererkrankungen (AKH Wien)

ERN ReCONNET (ERN für seltene und komplexe Bindegewebs- und muskuloskelettale Erkrankungen)

Zentrum für erbliche und autoimmune Bindegewebserkrankungen Innsbruck (Medizinische Universität Innsbruck)

ERN RITA (ERN für Immundefizienzen, autoinflammatorische und Autoimmunkrankheiten)

Universitätsklinik für Neurologie Graz (Medizinische Universität Graz)

Zentrum für autoinflammatorische Erkrankungen und Autoimmunopathien (Medizinische Universität Innsbruck)

Zentrum für Immundefekterkrankungen (AKH Wien/St. Anna Kinderspital Wien)

ERN TRANSPLANT-CHILD (ERN für Transplantation im Kindesalter)

Zentrum für pädiatrische Lungentransplantation (AKH Wien)

VASCERN (ERN für seltene vaskuläre Erkrankungen)

Zentrum für vaskuläre Anomalien im Kindesalter (Medizinische Universität Graz)

Zentrum für genetische Aortopathien (Medizinische Universität Innsbruck)

Abteilung für Kinder- und Jugendchirurgie (Sozialmedizinisches Zentrum Ost Wien)

ANZ

$\mathrm{ANZ}^{\mathrm{a}}$

$\mathrm{ANZ}^{\mathrm{a}}$

$\mathrm{ANZ}^{\mathrm{a}}$

$\mathrm{ANZ}^{\mathrm{a}}$

EZ

ANZ

ANZ

ANZ

ANZ

ANZ

ANZ

ANZ

ANZ

ANZ

EZ Expertisezentrum (Typ B-Zentrum), ANZ Assoziiertes Nationales Zentrum

${ }^{a} A N Z$, die sich aktuell in einem laufenden nationalen Designationsverfahren zu einem Expertisezentrum befinden

lich in praktisch allen Fällen nicht nur mehrere Bewerber pro ERN - dies ist schon in der derzeit laufenden dritten Designationsrunde der Fall -, sondern die Bewerber differieren untereinander in ihrem Leistungsportfolio (gemäß den Angaben aus den ANZ-Bewerbungsbögen und bezogen auf die Basisanforderungen des jeweiligen ERN) in einem Ausmaß, das in vielen, vermutlich sogar in allen Fällen eine Selektion derjenigen Einrichtungen erforderlich machen wird, die ein nachfolgendes Designationsverfahren erfolgreich bestehen kön- nen. Diese Entscheidungsfindung kann für betroffene engagierte Spezialisten und Einrichtungen schwierig sein, daher soll sie in einem gemeinsamen, transparenten und offenen Diskussionsprozess wie an einem Runden Tisch (daher Roundtable) unter Berücksichtigung aller Daten und Anforderungen erfolgen.

Die Roundtables sollen darüber hinaus auch als Anstoß dienen, in dieser nicht einfachen Situation die themenund fachgebietsbezogene Vernetzung der jeweiligen Einrichtungen in Österreich voranzutreiben und auf ein völlig neues
Kooperations- und Integrationsniveau zu heben, sodass am Ende, dem Vorbild der französischen „Filières de santé maladies rares" folgend, innerösterreichische $\mathrm{Re}$ ferenznetzwerke für die einzelnen Fachbereiche entstehen, die wirklich gelebt werden. So könnte auch gewährleistet werden, dass, wenn beispielsweise aufgrund einer mangelnden Erfolgsaussicht auf eine erfolgreiche nationale Designation oder aufgrund neuer Entwicklungen auf EU-Ebene nicht alle Mitglieder eines innerösterreichischen Referenznetzwerks auch Vollmitglieder im zugehöri- 


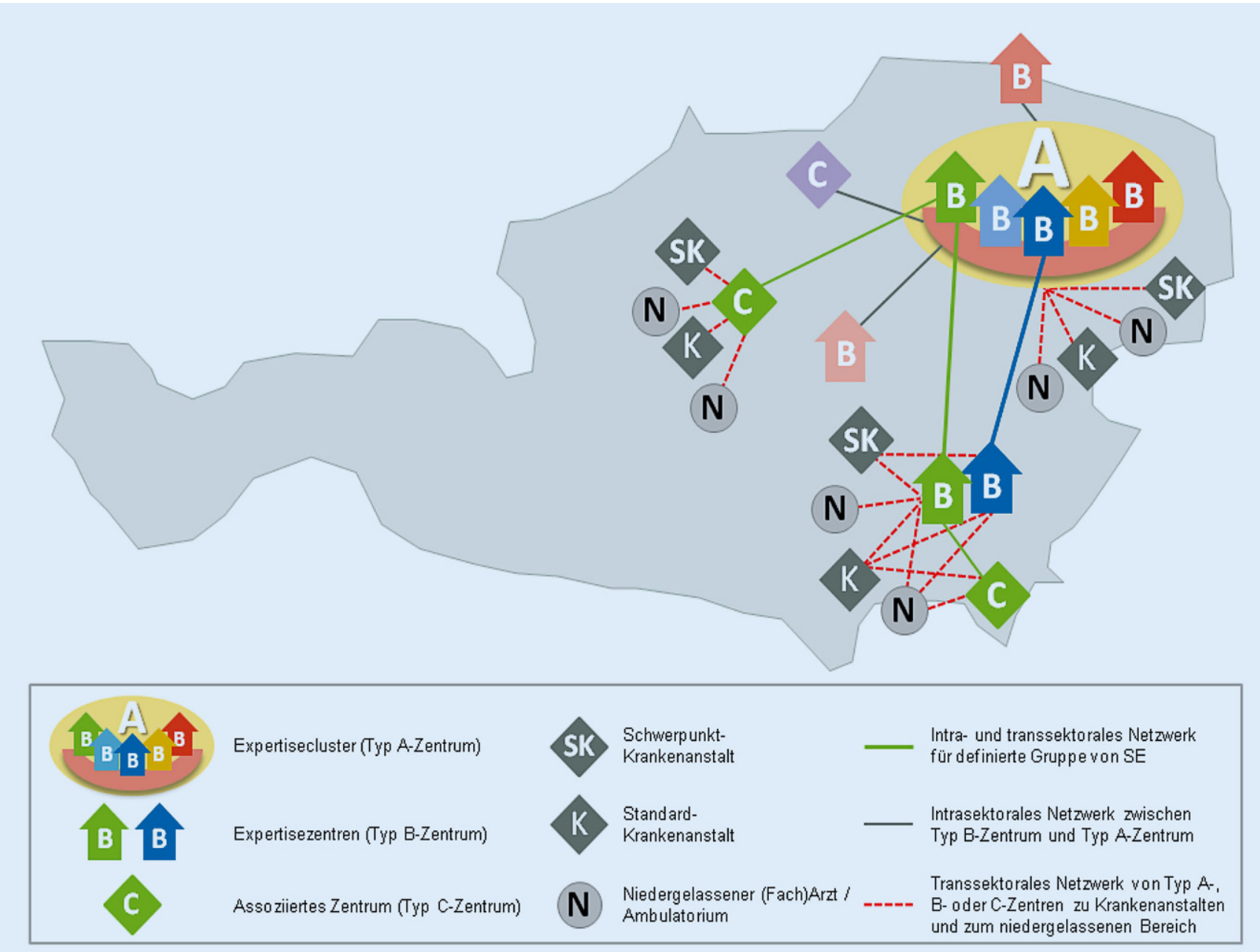

Abb. 1 \& Schematische Darstellung der nationalen transsektoralen Vernetzungsformen zwischen Typ A- und Typ B-Zentren auf der einen sowie Typ C-Zentren, Universitätskliniken, anderen Krankenanstalten und dem niedergelassenen Bereich auf der anderen Seite. SE seltene Erkrankungen. (Nach Ladurner und Vogtländer [3])

gen ERN werden können, dennoch alle Zentren eine strukturelle Anbindung an und indirekte Integration in das ERN haben. Denn in diesem Fall könnten jenes Partnerzentrum oder jene Partnerzentren, die Mitglieder in diesem ERN sind oder werden, als Brücke zwischen ERN und nationalem Referenznetzwerk fungieren, über die alle Inhalte des ERN an alle inländischen Partnerzentren weitergeleitet und umgekehrt auch die Interessen aller inländischen Partnerzentren im ERN vertreten werden. Dieses Gedankenkonzept folgt dem zentralen Credo bei der Konzeption der ERN, das bisherige System aus Konkurrenz und manchmal auch Konfrontation, von dem kein Patient und keine Patientin je profitierte, abzulösen durch ein System der Koordination und Kooperation.
Die Umstellung des derzeitigen ERNKonzepts auf ein Modell nationaler Themen- bzw. Fachgebiets-bezogener Referenznetzwerke mit ausgewählten Knotenpunkten in das jeweilige ERN wird angesichts völlig ausufernder Antragszahlen für neue Mitglieder in ERN inzwischen in allen europäischen Mitgliedstaaten auf breiter Ebene diskutiert. Und wenn man ehrlich ist, muss man zugeben, dass Europa, will es funktionsfähige ERN erhalten, nicht um diesen Transformationsschritt herumkommen wird. Je früher wir uns in Österreich also mit diesem Thema befassen, desto besser.

Ergänzt werden soll diese Designationsoffensive, so ist der aktuelle Plan, durch die Entwicklung und Ausarbeitung österreichweiter Informations- und Disseminationsstrategien - zum Zentren- modell im Allgemeinen und zu den konkreten Expertisezentren, den ANZ sowie den ERN im Speziellen. Denn jetzt, nachdem Österreich mit 49 Zentren in allen Netzwerken vertreten ist und über bislang 9 und bald 18 Expertisezentren (Typ B-Zentren) verfügen wird, ist der ideale Zeitpunkt gekommen, um all diese Zentren und ihre Expertiseschwerpunkte landesweit bekannt zu machen und $\mathrm{zu}$ bewerben und sie so möglichst nahtlos in die bestehende österreichische Versorgungslandschaft bis hinunter in den niedergelassenen Bereich der Haus- und Fachärztinnen und -ärzte zu integrieren (- Abb. 1). Diese Informationsoffensive wird, um sie möglichst effektiv gestalten zukönnen, überverschiedene Kanälelaufen müssen und ist letztlich eine Aufgabe aller Akteure in diesem Feld. 


\section{Handlungsfeld 1 - Abbildung seltener Erkrankungen im Gesundheitssystem}

Nachdem die Umsetzung des Zentrenkonzepts in vollem Gange ist, kann nun in HF 1 (•Tab. 1) jenes Maßnahmenpaket wieder aufgegriffen werden, dessen Umsetzung eine möglichst umfassende Kodierung von seltenen Erkrankungen in Österreich zum Ziel hat. Hierbei ist vorgesehen, in einem schrittweisen Prozess - zunächst in Expertisezentren (Typ B-Zentren) und anschließend in ANZ - die ergänzende Verwendung der sogenannten Orpha-Codes (eines für die Orphanet-Datenbank entwickelten, sehr differenzierten und praktisch alle Krankheitsbilder im Bereich der seltenen Erkrankungen abdeckenden Kodierungssystems) in der Diagnosenund Leistungsdokumentation der Kliniken (parallel zum System der ICD-10Kodierung) einzuführen. Die inhaltlichen Vorarbeiten hierzu, insbesondere die passende Synchronisierung der ICD10- und Orpha-Codes in einem beide Kodierungsebenen verbindenden elektronischen Masterfile, wurden auf europäischer Ebene im Rahmen eines EUProjekts (Joint Action „RD-Action“) bereits 2018 abgeschlossen. In Österreich wäre es jetzt erforderlich, die Inhalte dieses Masterfiles in einer nutzerfreundlichen und einfach anzuwendenden Form, ohne signifikanten Mehraufwand für das Klinikpersonal, in die jeweiligen Krankenhausinformationssysteme jener Spitäler und Kliniken zu integrieren, in deren Wirkungskreis Expertisezentren und ANZ angesiedelt sind. Diese Arbeiten sollen schrittweise per Standort erfolgen, beginnend mit ein oder zwei Expertisezentren, die sich freiwillig als Piloteinrichtungen zur Verfügung stellen.

\section{Handlungsfeld 3 - Verbesserung der Diagnostik von seltenen Erkrankungen}

In HF 3 (-Tab. 1) sollen die bereits begonnenen und weit fortgeschrittenen Arbeiten im Zusammenhang mit einer Verbesserung der Qualitätssicherung in der Labordiagnostik fortgesetzt und $\mathrm{zu}$ einem ersten Abschluss gebracht werden. Konkret geht es dabei um die
Wiederaufnahme und Fortsetzung der Arbeiten am Konzept der Expertiselabors, in dem - analog zum Modell der Typ A-, Typ B- und Typ C-Zentren in Anhang 3 des NAP.se - ein Leistungs- und Qualitätsstandard für jene hochspezialisierten Laboreinrichtungen definiert werden soll, die spezielle diagnostische Untersuchungen für seltene Erkrankungen anbieten. Im Unterschied $\mathrm{zu}$ den klinischen Einrichtungen, für die derartige Standards aus den diversen europäischen Quellen erst zusammengestellt und definiert werden mussten, existiert im Laborbereich mit der medizinisch ausgerichteten Akkreditierungsnorm ISO 15189 bereits ein international etabliertes Qualitätssystem und Prüfverfahren, das mit wenigen Ergänzungen für ein Designationsverfahren für Expertiselabors verwendet werden kann, sodass es hierfür keiner völligen Neuentwicklung in Österreich bedarf. Nachdem es 2018 zunächst einzelne politische Bedenken hinsichtlich des Kosten-NutzenVerhältnisses dieser Maßnahme gab, steht der Anwendung der ISO-15189Norm für ein Designationsverfahren für Expertiselabors inzwischen nichts mehr im Weg, da die Republik Österreich im Rahmen der Umsetzung der In-vitro-Diagnostika-Richtlinie [8] ohnedies verpflichtet ist, alle Laboreinrichtungen gemäß ISO 15189 oder einer vergleichbaren Akkreditierungsnorm zu akkreditieren.

\section{Zukunftsperspektiven 2 \\ - Gedankenspiele auf \\ Basis bisheriger nationaler \\ Überlegungen und rezenter \\ europäischer Entwicklungen}

\section{Handlungsfeld 2 - Medizinisch- klinische Versorgung}

Mögliche mittelfristige Zukunftsperspektiven im Bereich von HF 2 umfassen 3 Aktivitäten. Zwei davon besitzen eine sehr hohe Umsetzungswahrscheinlichkeit, da sie eine Fortschreibung der Umsetzung des Zentrenmodells und damit einen zentralen Inhalt des NAP.se darstellen. Gemeint sind hier - nach Abschluss der ersten, umfassenden Welle der Expertisezentren (Typ B-Zentren) - die Designation von Assoziierten Zentren
(Typ C-Zentren) und die Entwicklung konkreter operationaler Kriterien und eines darauf zugeschnittenen Designationsverfahrens für Expertisecluster (Typ A-Zentren). Denn während für das weitere Vorgehen bei Typ C-Zentren schon viele Dokumente und Verfahrensschritte durch die Entwicklung und Ausarbeitung des Designationsverfahrens für Typ B-Zentren - und die erste Adaptierung dieser Unterlagen für den nationalen Bewerbungsprozess zur Teilnahme als ANZ an dem zugehörigen ERN - vorbereitet sind und nur an die etwas anders gelagerten inhaltlichen, strukturellen und organisatorischen Anforderungen des Typ C-Zentrums angepasst werden müssen, existieren für Typ A-Zentren bislang nur die in Anhang 3 des NAP.se beschriebenen allgemeinen Leistungsund Qualitätskriterien [4]. In ersten, noch vorläufigen Planungsüberlegungen ist angedacht, bei der Ausarbeitung der konkreten operationalen Kriterien für Expertisecluster möglichst viele der bis dahin gewonnenen Erfahrungen mit existierenden, zumeist selbsternannten, multidisziplinären SE-Zentren in Österreich, aber beispielsweise auch in Deutschland, zu berücksichtigen.

Neben diesem Typ A- und Typ CSchwerpunkt wird es bei Bedarf und entsprechender Beschlusslage auf der politischen Planungsebene auch weitere Designationsverfahren für Expertisezentren (Typ B-Zentren) für jene Einrichtungen geben, die ihr Interesse an einem Designationsverfahren erst später, d.h. nach der eingangs geschilderten ersten Runde der Interessenbekundungen (• Tab. 2), angemeldet haben.

Die dritte mögliche Aktivität im Bereich von HF 2 ist ein neuer Gedanke, der nicht im NAP.se verankert ist, sondern aus Entwicklungen auf europäischer Ebene geboren wurde. Dort wurde mit Etablierung der ersten 24 ERN im März 2017 auch ein Gremium der Netzwerkkoordinatoren (die sogenannte ERN Coordinators Group) eingerichtet, das seither erfolgreich in verschiedenen Arbeitsgruppen gemeinsame Aktivitäten vorantreibt, die für alle ERN gleichermaßen relevant sind. Nach diesem Vorbild wäre die Einrichtung eines primär virtuell operierenden Nationalen Gremi- 
ums der Zentrumskoordinatoren anzudenken, das sich initial aus den Koordinatorinnen bzw. Koordinatoren der aktuellen Typ B-Zentren und ANZ (und später dann aus denjenigen der dann designierten Typ B- und Typ C-Zentren) zusammensetzt, ergänzt um jene Patientinnen- und Patientenvertreter aus Österreich, die als sogenannte ePAG's aktiv an der Arbeit der jeweiligen ERN teilnehmen. Auch die Einbeziehung weiterer zentraler Systempartner könnte angedacht werden. Dieses Gremium, das unter dem Vorsitz des Gesundheitsministeriums stünde, würde nicht nur allen Zentrumskoordinatoren eine Plattform für den regelmäßigen strukturierten Austausch mit den Gesundheitsbehörden bieten und somit auch ihre Leistungen im Feld der seltenen Erkrankungen hervorheben, es wäre auch eine ideale Plattform, um übergeordnete Fragestellungen im Kontext der ERN-Arbeit wie auch im Kontext der nationalen Zentrenarbeit zu diskutieren und so die gesundheitspolitische Ebene mit Informationen $\mathrm{zu}$ versorgen, die dann in den Diskussionsprozess mit den anderen Mitgliedstaaten eingebracht werden könnten. Natürlich sind auch weitere Funktionen denkbar, und anlassbezogen könnten und sollten weitere Akteure einbezogen werden. Erste informelle Vorgespräche zur Einrichtung eines derartigen Gremiums hat es auf politischer Ebene bereits gegeben, weiter ist diese Perspektive aber bislang noch nicht gereift.

\section{Handlungsfeld 1 - Abbildung seltener Erkrankungen im Sozialsystem}

In HF 1 wäre als mittelfristige Zukunftsperspektive an eine Maßnahme zu denken, die der NAP.se bislang nur sehr kursorisch anspricht, und zwar die Überbrückung der unterschiedlichen Klassifikationssysteme im Gesundheits- und Sozialbereich. Während der Gesundheitsbereich nämlich ein krankheitsorientiertes Klassifikationssystem verwendet (aktuell die ICD 10 [International Classification of Diseases, Version 10] in der letzten Fassung von 2019), kommt im Sozialbereich ein funktionsorientiertes Klassifikationssystem (die ICF [International Classification of Functioning, Disability and Health] in der letzten Fassung von 2005) zur Anwendung. Ohne Bindeglied sind diese beiden Systeme nicht interoperabel, ein Problem, dass sich besonders im Bereich der seltenen Erkrankungen stellt, da hier - aufgrund der häufig multisystemischen und damit viele Funktionsbereiche gleichzeitig betreffenden Erkrankungen - funktionelle Defizite in einem Bereich meist eine gravierendere Auswirkung haben, da sie durch zusätzliche parallele Einschränkungen in anderen Bereichen weniger abgemildert werden können als es bei Patientinnen und Patienten der Fall wäre, die nur unter einem Defizit leiden. Eine mögliche und relativ einfach für Österreich zu adaptierende Brücke würde hier das seit mehreren Jahren von der Datenbank Orphanet gestaltete Projekt „Orphanet disability project“ bieten, in dem für inzwischen 857 seltene Erkrankungen (Stand Ende 2019) spezifische Behinderungsdatensätze und begleitende, medizinisch validierte Erläuterungen entwickelt wurden, die von der ICF-Klassifikation abgeleitet wurden und damit vollständig kompatibel zu dieser Klassifikation sind [9]. Diese Datensätze und begleitenden Erläuterungen müssten in einem ersten Schritt auf ihre Verwendbarkeit im österreichischen System hin analysiert werden. Sollte diese Prüfung erfolgreich verlaufen, sodass keine oder keine umfangreichen Anpassungen erforderlich wären, könnte diese Maßnahme im Prinzip sehr einfach und rasch durch eine entsprechend adaptierte Übersetzung der bereits existierenden Informationen und Datensätze vorangetrieben werden.

\section{Zukunftsperspektiven 3 -} Eine Wunschliste für weitere, längerfristige Entwicklungsziele des NAP.se

Die im Folgenden kurz angerissenen, länger- bis langfristigen möglichen $\mathrm{Zu}$ kunftsperspektiven bei der Umsetzung und Weiterentwicklung des NAP.se fallen in den Bereich der HF 1, HF 2 und HF 4 sowie neu zu definierender HF. Sie stellen ausgewählte präliminäre Vorschläge ohne Anspruch auf Vollständigkeit dar. Angeregt wurden sie entweder durch die Beobachtung sehr überzeugender
Aktivitäten und Konzepte in anderen europäischen Ländern oder durch im NAP.se festgelegte Maßnahmen, deren Umsetzung erst in der Endphase der Gesamtimplementierung des Aktionsplans vorgesehen ist. Ob bzw. wann es zu einer Verwirklichung dieser Gedanken kommt, wurde auf der gesundheitspolitischen Ebene noch nicht diskutiert und entschieden.

\section{Einführung eines holistischen Case-Management-Systems in Österreich}

Österreich gehört zu jenen Ländern, in denen der Begriff eines Case Managers bzw. einer Case Managerin bislang noch nicht klar definiert wurde, geschweige denn als eigenes Berufsbild festgelegt und anerkannt wäre. Folgerichtig findet sich in den bislang vom NB-NAP.se bearbeiteten Anträgen auf Anerkennung als Expertisezentrum eine Vielzahl unterschiedlich strukturierter und detaillierter Konzepte unter Einbeziehung verschiedenster Personalebenen, wobei nicht selten wertvolle Zeitressourcen bei Ärztinnen und Ärzten gebunden werden. Und ungeachtet des unbestrittenen Engagements all der Kolleginnen und Kollegen kommt diese Koordinationstätigkeit an ihre Grenzen, sobald der rein medizinische Bereich verlassen wird. So wandert die Koordinationsverantwortung fast immer von einem Bereich zum anderen, was zwangsläufig zu Verzögerungen, Ineffizienzen und gegebenenfalls einem „Verlust" des Patienten bzw. der Patientin führt, der bzw. die sich im System verloren fühlt und aufgibt - alles zusammengenommen das Gegenteil einer vorbildhaften, umfassenden und effizienten Fallkoordination, die die Betroffenen auch in ihrer Eigenverantwortung und ihren eigenen Handlungsmöglichkeiten unterstützen und stärken soll.

Nun kann man die durchaus berechtigte Frage stellen, ob ein Expertisezentrum wirklich der beste Ort für diesen holistischen Begleitansatz für Patienten und Patientinnen und allfällige Familienangehörige ist, so wie es die Empfehlungen des EUCERD vorsahen [5]. Die zwangsläufige Gegenfrage wäre allerdings: Welcher Ort wäre es dann und hier lohnt ein Blick über den ös- 
terreichischen Gartenzaun hinweg nach Norwegen, auf das Konzept der sogenannten Resource Centres [10], die einen ganz anderen, dennoch exemplarischen Lösungsansatz bieten. Denn in einem Resource Centre kommen Patientinnen und Patienten und Familienangehörige mit Erkrankungen, die der gleichen oder von den Anforderungen her einer vergleichbaren Krankheitsgruppe angehören, einmal über einen Zeitraum von 14 Tagen in einer für die verschiedensten medizinischen Anforderungen ausgestatteten Einrichtung zusammen, die alle erforderlichen pflegerischen, therapeutischen und rehabilitativen Möglichkeiten bietet, um sich vollumfassend zu allen gesundheitlichen, sozialen und familiären Aspekten ihrer Erkrankung sowie den dafür verfügbaren staatlichen und anderen Unterstützungsmöglichkeiten $\mathrm{zu}$ informieren und zu verstehen bzw. zu lernen, welche eigenen Schritte zur Erlangung all dieser Unterstützungen erforderlich sind und wie man sie in Angriff nimmt. Diese Form des Empowerment der Patientinnen, Patienten und Familien im Umgang mit ihrer Erkrankung - in konzentrierter Form zu einem Zeitpunkt an einem Ort im Sinn des One-Stop-Shop-Modells vermittelt - mag zunächst aufwendig erscheinen, führt aber, wie Ergebnisse u. a. des EU-Projekts „InnovCare“ nahelegen [11], letztlich $\mathrm{zu}$ einem besseren Umgang der Betroffenen mit ihrer Erkrankung und zu einer teils deutlich positiveren Bewertung ihrer Lebenssituation und-unterstützung, was ihnen wiederum mehr Kraft zur Bewältigung der vielen Anforderungen verleiht [12].

Eine De-novo-Einrichtung derartiger Resource Centres in Österreich wäre illusorisch, ist aber vielleicht auch gar nicht nötig. Stattdessen sollte man z.B. über die Möglichkeiten zu einer engen, strukturellen Kooperation von geeigneten $\mathrm{Re}$ ha-Zentren und Expertisezentren (deren Zahl ja weiter steigen wird) nachdenken, die beide jeweils für ihren Bereich große Teile der notwendigen Kernexpertise mitbringen, die derartige Resource Centres auszeichnet. Und Reha-Zentren wären auch die idealen Einrichtungen, um Betroffene und Familienangehörige für 2-wöchige Intensivschulungen mit begleitender hochspezialisierter Betreuung aufnehmen zu können. Natürlich müssten dann noch entsprechende Expertisen für den sozialen und den (familien-)psychologischen Bereich hinzukommen, doch auch dafür ließen sich sicher geeignete Institutionen und Formen finden.

\section{Evaluationskonzept für designierte österreichische Zentren}

Der NAP.se sieht eine Evaluation designierter Zentren, seien es Expertisezentren (Typ B-Zentren), Assoziierte Zentren (Typ C-Zentren) oder Expertisecluster (Typ A-Zentren), 5 Jahre nach ihrer Designation vor, um bei einem positiven Evaluationsergebnis die jeweilige Designation um weitere 5 Jahre zu verlängern. Nachdem das nationale Designationsverfahren zunächst mit Typ BZentren begonnen hat, ist es auch dieser Zentrumstyp, für den in wenigen Jahren pilotmäßig ein Evaluationskonzept erarbeitet und umgesetzt werden muss. Um dieses Verfahren so wenig aufwendig und zugleich so evidenzbasiert wie möglich zu gestalten, wird es u. a. darauf ankommen, geeignete, d.h. für die Funktion des jeweiligen Zentrums aussagekräftige, und relativ einfach messbare Indikatoren $\mathrm{zu}$ entwickeln, wobei parallele Prozesse auf internationaler Ebene, wie beispielsweise die Entwicklung geeigneter Indikatoren auf EU-Ebene, die ein kontinuierliches Monitoring und eine anschließende Evaluation der Arbeiten und Leistungen der ERN ermöglichen sollen, unbedingt berücksichtigt werden sollten.

\section{Fazit für die Praxis}

Wie in vielen anderen Lebensbereichen auch, mahlen die Mühlen bei der Umsetzung des NAP.se langsam. Aber so manchen Unkenrufen zum Trotz, die keine Fortschritte sehen wollen, ist bereits einiges geschehen. Was vor wenigen Jahren noch weitgehend undenkbar schien - eine klar gegliederte und gut sichtbare Versorgungslandschaft geprüfter spezialisierter Zentren für seltene Erkrankungen - ist aktuell schon weit gediehen und wird in den kommenden 2-3 Jahren seinen vollen Umfang erreichen und damit sein volles Potenzial entfalten. Dann kann auch die Einrichtung der Expertisecluster (Typ A-Zentren) als nächste Stufe der Integration von Expertise in ein übergeordnetes Gesamtkonzept in Angriff genommen werden.

Essenziell wird darüber hinaus die weiter zu optimierende, gelebte Einbindung all dieser Zentren in die existierende österreichische Versorgungslandschaft sein, ein Vorhaben, dessen Erfolg nicht nur von der im Beitrag angesprochenen, über diverse Kanäle zu lancierenden Informationsoffensive abhängen wird, sondern auch von der ebenfalls oben angesprochenen erfolgreichen Einrichtung fach- bzw. themengebietsbezogener nationaler Referenznetzwerke aller spezialisierten Einrichtungen innerhalb eines übergeordneten Themen- oder Fachbereichs. Und während aktuell die erste Umsetzungsphase des Zentrenmodells noch in vollem Gange ist, öffnen sich jetzt für viele weitere Maßnahmen im NAP.se, die zunächst zurückgestellt worden waren, da sie von einer vorangehenden Zentren-Designation abhängig waren, die Aussichten auf eine zeitnahe, wenn auch weiterhin in kleinen Schritten erfolgende Realisierung.

Der NAP.se, speziell in seiner Kombination aus Plan und Strategie, hat sein Potenzial noch bei Weitem nicht ausgeschöpft und bietet sich - ungeachtet aller nach wie vor bestehenden Probleme im Bereich der seltenen Erkrankungen - auch weiterhin als das geeignetste Instrument an, weitere Lösungsansätze zu entwickeln und umzusetzen. Allerdings ist hierfür die Unterstützung aller Akteure essenziell - es kommt auf jeden einzelnen von uns an. 


\begin{tabular}{ll}
\hline Korrespondenzadresse \\
Assoc.-Prof. PD Dr. \\
Till Voigtländer \\
Universitätsklinik für \\
Neurologie, Abteilung \\
für Neuropathologie und \\
Neurochemie, Medizinischer \\
Universitätscampus Wien \\
und Nationales Büro \\
für die Umsetzung und \\
Weiterentwicklung des \\
NAP.se, Medizinische \\
Universität Wien \\
Währinger Gürtel 18-20, \\
1090 Wien, Österreich \\
till.voigtlaender@ \\
meduniwien.ac.at
\end{tabular}

Assoc.-Prof. PD Dr. Till Voigtländer Studium der Humanmedizin an der Ruprecht-Karls-Universität Heidelberg, Dissertation an der Freien Universität Berlin, Teilausbildung zum Facharzt für Neuropathologie (Zürich, Wien), Ausbildung zum Facharzt für Neurobiologie (Wien), Habilitation an der Medizinischen Universität Wien. Mehrere Studienaufenthalte an der St. Louis University School of Medicine/Boston University. Zurzeit assoziierter Professor in der Abteilung für Neuropathologie und Neurochemie der Universitätsklinik für Neurologie, Medizinischer Universitätscampus Wien. Arbeitsschwerpunkte im Bereich seltene Erkrankungen: ab 2004 Länderkoordinator für Orphanet in Österreich; 2008-2010 Leiter der Unterkommission für seltene Erkrankungen des Obersten Sanitätsrats; 2011-2018 medizinischer Leiter der Nationalen Koordinationsstelle für seltene Erkrankungen (NKSE) an der Gesundheit Österreich GmbH (GÖG); seit 2019 Leiter des Nationalen Büros für die Umsetzung und Weiterentwicklung des NAP.se (NBNAP.se); ab 2011 österreichischer Vertreter in diversen Gremien der Europäischen Kommission zum Thema seltene Erkrankungen (European Union Committee of Experts on Rare Diseases, Cross Border Healthcare Directive Expert Group, Commission Expert Group on Rare Diseases); ab 2014 Mitglied und Mitvorsitzender (2014-2018) des Beschlussorgans der Mitgliedsstaaten für die Europäischen Referenznetzwerke (Board of Member States on ERN). 2019 Auszeichnung mit dem "Black Pearl European Rare Disease Leadership Award" der Organisation EURORDIS

Funding. Open access funding provided by Medical University of Vienna.

\section{Einhaltung ethischer Richtlinien}

Interessenkonflikt. T. Voigtländer ist Leiter des Leiter des Nationalen Büros für die Umsetzung und Weiterentwicklung des NAP.se (NB-NAP.se) an der Universitätsklinik für Neurologie der Medizinischen Universität Wien, das vom Bundesministerium für Soziales, Gesundheit, Pflege und Konsumentenschutz mit der Koordination und Umsetzung sowie Weiterentwicklung des Nationalen Aktionsplans für Seltene Erkrankungen (NAP.se) beauftragt ist.

Für diesen Beitrag wurden vom Autor keine Studien an Menschen oder Tieren durchgeführt. Für die aufge- führten Studien gelten die jeweils dort angegebenen ethischen Richtlinien.

Open Access. Dieser Artikel wird unter der Creative Commons Namensnennung 4.0 International Lizenz veröffentlicht, welche die Nutzung, Vervielfältigung, Bearbeitung, Verbreitung und Wiedergabe in jeglichem Medium und Format erlaubt, sofern Sie den/die ursprünglichen Autor(en) und die Quelle ordnungsgemäß nennen, einen Link zur Creative Commons Lizenz beifügen und angeben, ob Änderungen vorgenommen wurden.

Die in diesem Artikel enthaltenen Bilder und sonstiges Drittmaterial unterliegen ebenfalls der genannten Creative Commons Lizenz, sofern sich aus der Abbildungslegende nichts anderes ergibt. Sofern das betreffende Material nicht unter der genannten Creative Commons Lizenz steht und die betreffende Handlung nicht nach gesetzlichen Vorschriften erlaubt ist, ist für die oben aufgeführten Weiterverwendungen des Materials die Einwilligung des jeweiligen Rechteinhabers einzuholen.

Weitere Details zur Lizenz entnehmen Sie bitte der Lizenzinformation auf http://creativecommons.org/ licenses/by/4.0/deed.de.

\section{Literatur}

1. Kommission der europäischen Gemeinschaften (2008) Mitteilung der Kommission an den Rat das Europäische Parlament, den Europäischen Wirtschafts- und Sozialausschuss und den Ausschuss der Regionen über seltene Krankheiten - eine Herausforderung für Europa. KOM(2008) 679 endgültig. Kommission der europäischen Gemeinschaften, Brüssel, S2-12

2. Rat der Europäischen Union (2009f) Empfehlung des Rates vom 8. Juni 2009 für eine Maßnahme im Bereich seltener Krankheiten (2009/C 151/02). Amtsblatt der Europäischen Union C 151, S7-10

3. Ladurner J, Voigtländer T (2015) Nationale und europäische Konzepte zur Bündelung der Expertise für seltene Erkrankungen. Teil 1: Der Nationale Aktionsplan für seltene Erkrankungen (NAP.se). Paediatr Paedolog 50(Suppl 2):66-73

4. Bundesministerium für Gesundheit (2015) Nationaler Aktionsplan für seltene Erkrankungen NAP.se/2014-2018. Erstellt von der Nationalen Koordinationsstelle für seltene Erkrankungen (NKSE) im Auftrag des Bundesministerium für Gesundheit. Bundesministerium für Gesundheit, Wien, S1-111

5. European Union Committee of Experts on Rare Diseases (2011) EUCERD recommendations - quality criteria for centres of expertise for rare diseases in member states. European Union Committee of Experts on Rare Diseases, Luxembourg, S2-12

6. Voigtländer T, Ladurner J (2015) Nationale und europäische Konzepte zur Bündelung der Expertise für seltene Erkrankungen. Teil 2: Konzentration und Vernetzung in Europa: Expertisezentren und Referenznetzwerke. Paediatr Paedolog 50(Suppl 2):74-80

7. Orphanet Austria (2019) Österreichische Mitglieder in Europäischen Referenznetzwerken (ERN). http://www.orpha.net/national/AT-DE/ index/startseite/.Zugegriffen:20. Juni 2020

8. Europäische Kommission (2017) Verordnung (EU) 2017/746 des Europäischen Parlaments und des Rates vom 5. April 2017 über In-vitro-Diagnostika und zur Aufhebung der Richtlinie 98/79/EG und des Beschlusses 2010/227/EU der Kommission. Amtsblatt der Europäischen Union L 117, S 176-332

9. Orphanet (2020) Das Portal für seltene Krankheiten und Orphan Drugs. Disability help section: information on activity limitation/participation restriction (functional consequences) described in rare diseases. https://www.orpha.net/consor/cgibin/Disease_Disability.php?lng=EN. Zugegriffen: 20. Juni 2020

10. INNOVCare (2020) Resource centres for rare diseases. https://innovcare.eu/social-services/ resource-centres-for-rare-diseases/. Zugegriffen: 20. Juni 2020

11. INNOVCare (2018) Results of the EU-funded project INNOVCare. https://innovcare.eu/bridging-thegaps-between-health-and-social-care-resultsof-the-eu-funded-project-innovcare-2018/. Zugegriffen:20. Juni 2020

12. EURORDISRare Diseases Europe (2019) Position paper:achieving holistic person-centred care toleave no one behind. http://download2.eurordis.org/ positionpapers/Position\%20Paper\%20Holistic \%20Care\%20for\%20Rare\%20Diseases_Final.pdf. Zugegriffen: 20. Juni 2020

Hinweis des Verlags. Der Verlag bleibt in Hinblick auf geografische Zuordnungen und Gebietsbezeichnungen in veröffentlichten Karten und Institutsadressen neutral. 